\title{
No todo es culpa de la anestesia: neumoencéfalo vs ictus tras anestesia neuroaxial.
}

Bardisa de la Iglesia B, Miñana Moll A, Ivars Párraga C, Soldado Matoses MA, Machado Ortiz S.

Hospital Francesc de Borja. Gandía, Valencia.

\section{Resumen}

Aunque las complicaciones neurológicas asociadas a las técnicas neuroaxiales de anestesia son muy poco frecuentes la potencial gravedad de las mismas constituye un motivo de preocupación tanto para los pacientes como para los profesionales.

La aparición de una alteración neurológica en el período postoperatorio tiende a interpretarse como yatrogénica, lo que en algunos casos puede dificultar el correcto diagnóstico.

Presentamos el caso de una paciente sometida a técnica combinada epidural-subaracnoidea, que presentó clínica de déficit neurológico focal a las 24 horas de la intervención. En la TAC craneal destacaba como hallazgo radiológico mínimos focos de neumoencéfalo, lo cual hizo que se atribuyera erróneamente la sintomatología a una complicación de la anestesia. En una TAC de control posterior se observó una región isquémica compatible con la clínica neurológica.

Ante un evento neurológico adverso en el postoperatorio, es imprescindible realizar una evaluación cuidadosa del mismo, valorando todas las posibles causas antes de etiquetarlo como yatrogénico.

\section{Introducción}

Aunque las complicaciones neurológicas asociadas a las técnicas neuroaxiales de anestesia son muy poco frecuentes la potencial gravedad de las mismas constituye un motivo de preocupación tanto para los pacientes como para los profesionales.

La aparición de una alteración neurológica en el período postoperatorio tiende a interpretarse como yatrogénica, lo que en algunos casos puede dificultar el correcto diagnóstico.

Presentamos el caso de una paciente sometida a técnica combinada epiduralsubaracnoidea, que presentó clínica de déficit neurológico focal a las 24 horas de la intervención. En la TAC craneal destacaba como hallazgo radiológico mínimos focos de neumoencéfalo, lo cual hizo que se atribuyera erróneamente la sintomatología a una complicación de la anestesia. En una TAC de control posterior se observó una región isquémica compatible con la clínica neurológica.

Ante un evento neurológico adverso en el postoperatorio, es imprescindible realizar una evaluación cuidadosa del mismo, valorando todas las posibles causas antes de etiquetarlo como yatrogénico.

\section{Caso Clínico}

Paciente octogenaria, con antecedentes de alergia a la morfina e insuficiencia venosa periférica, que ingresó en nuestro centro de forma programada para segundo tiempo quirúrgico de recambio de prótesis total de rodilla infectada. El estudio preoperatorio incluyó ECG, hemograma, bioquímica, 
pruebas de coagulación y radiografía de tórax que no mostraron alteraciones significativas.

Las intervenciones previas, tanto la implantación de la prótesis como el primer tiempo del recambio, se llevaron a cabo bajo anestesia neuroaxial sin incidencias destacables. La paciente recibía tratamiento tromboprofiláctico con bemiparina sódica a dosis de 3500 UI al día que se interrumpió 24 horas antes de la intervención.

Después de valorar el caso, el anestesiólogo responsable decidió realizar la intervención bajo técnica combinada epidural-subaracnoidea y la paciente se trasladó al quirófano. El catéter epidural se insertó sin incidencias en L2-L3 mediante técnica de pérdida de resistencia con aire y aguja de Tuhoy, con prueba de aspiración y dosis test negativas.

Seguidamente se realizó punción subaracnoidea en L3-L4 con aguja espinal $25 \mathrm{G}$ tipo Withacre $\mathrm{y}$ tras comprobar reflujo adecuado de líquido cefalorraquídeo, se inyectaron $3 \mathrm{ml} \mathrm{de}$ bupivacaína hiperbárica al $0,5 \%$ obteniendo un nivel anestésico en T12.

La intervención se prolongó por espacio de 2 horas y 30 minutos, durante las cuales la paciente presentó estabilidad hemodinámica $\mathrm{y}$ respiratoria. $\mathrm{Se}$ administraron bolos periódicos de midazolam para mantener una sedación consciente. Durante los últimos 30 minutos de la intervención, fue necesario administrar un bolo epidural de $5 \mathrm{ml}$ de lidocaína al $2 \%$ por disminución del nivel anestésico.

Finalizada la intervención, la paciente se trasladó al área de recuperación postanestésica donde se inició perfusión epidural de ropivacaína al $0,2 \%$ a 8 $\mathrm{ml} / \mathrm{h}$. Durante su estancia en recuperación la paciente se mantuvo clínicamente estable, asintomática y con una puntuación de $0 / 10$ en la escala visual analígica de dolor (EVA) por lo que pudo darse de alta a sala de hospitalización.

En la sala se continuó la perfusión epidural de ropivacaína y se inició el protocolo de tromboprofilaxis con bemiparina sódica $3500 \mathrm{UI} / 24$ horas. A las 24 horas de la intervención, la paciente presentó de forma brusca un cuadro de hemiparesia y hemihipoestesia izquierda con desviación de la comisura bucal, disartria y desorientación, por lo que se avisó al servicio de anestesia. Ante tal situación se solicitó TAC urgente, en el que se informó de la presencia de neumoencéfalo subaracnoideo en cisternas peritronculares y frontal izquierdo sin otras alteraciones significativas (fig 1) sugiriéndose la punción raquídea como origen del mismo. Consultado el caso con el servicio de neurocirugía del hospital de referencia, se decidió manejo conservador y realizar TAC de control en 48-72 horas.

El catéter epidural se retiró respetando la ventana de seguridad de 12 horas entre dosis consecutivas de bemiparina. La paciente se mantuvo estable y tras el deterioro inicial progresivamente comenzó una recuperación del déficit neurológico. 


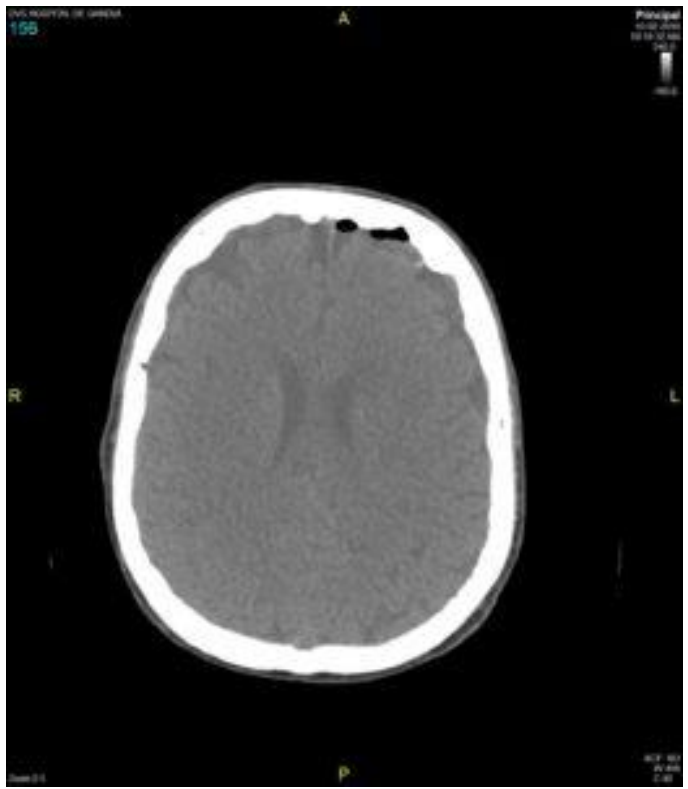

Fig. 1: TAC inicial. Se aprecian pequeñas áreas de neumoencéfalo subaracnoideo en región frontal izquierda

En la TAC de control realizada a las 48 horas del evento se evidenció pequeña área hipodensa cortico-subcortical frontoparietal derecha de nueva aparición (fig 2 y 3), compatible con infarto agudo en territorio de arteria cerebral media derecha, junto con evidente mejoría del neumoencéfalo, quedando tan solo alguna burbuja frontal izquierda.

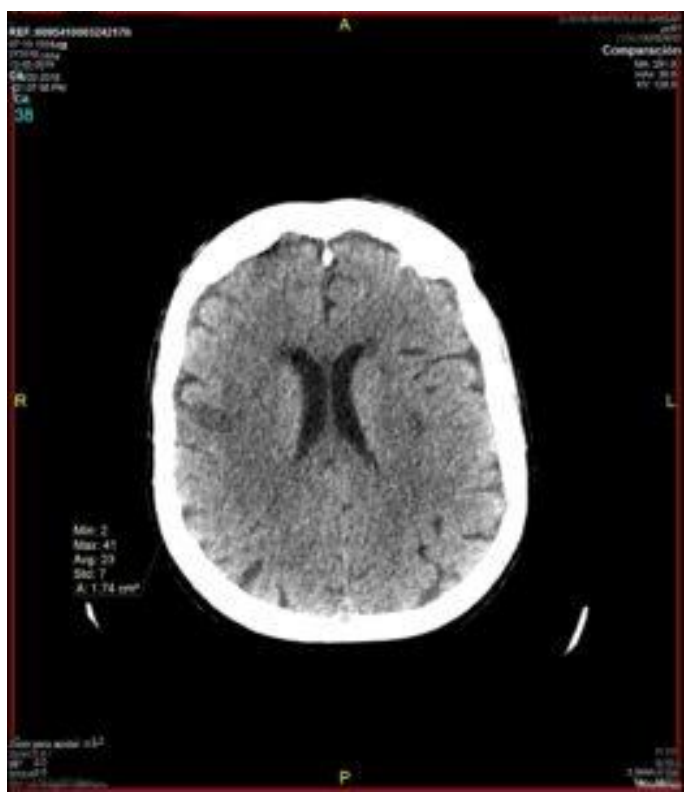

Fig. 2: TAC de control a las 48 horas. Se aprecia área isquémica frontoparietal derecha de nueva aparición, junto con resolución del neumoencéfalo

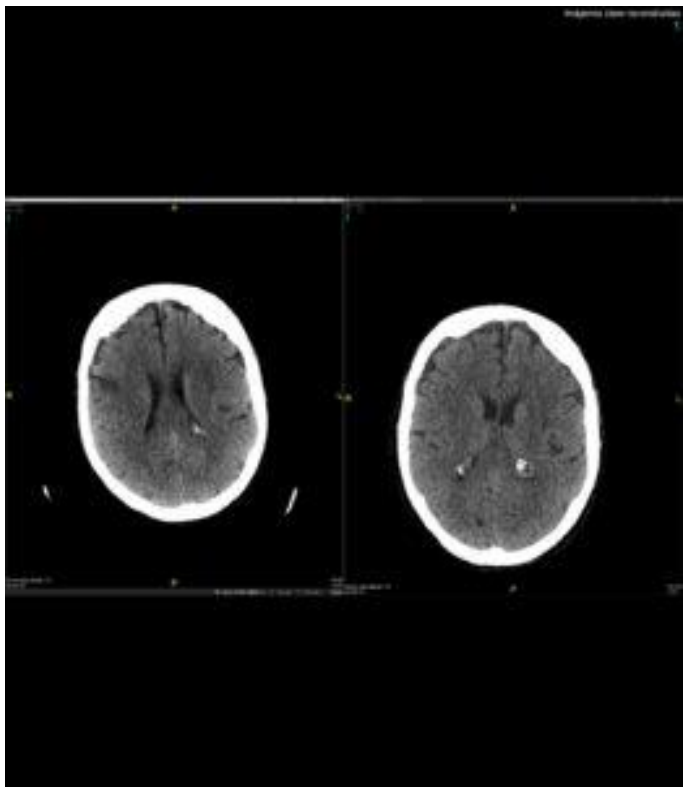

Fig. 3: Comparativa entre TAC inicial (derecha) y el de control (izquierda), donde se aprecia la aparición de área isquémica

Se completó el diagnóstico etiológico con doppler carotídeo que no mostró alteraciones significativas. Se inició tratamiento con AAS por parte de medicina interna, y ocho días tras la intervención la paciente fue dada de alta a domicilio para proseguir rehabilitación en régimen ambulatorio.

Como secuela del ictus, al alta persistía ligera disminución de fuerza en hemicuerpo izquierdo. En la visita de control a las 5 semanas postoperatorias, la evolución había sido satisfactoria tanto en lo referente al cuadro neurológico del que se encontraba asintomática, como en lo referente a la función articular.

\section{Discusión}

El neumoencéfalo como complicación de las técnicas neuroaxiales es un evento raro y la mayor parte de casos publicados están en relación con la punción dural accidental durante la localización del espacio epidural mediante técnica de pérdida de resistencia con aire, por lo que algunos autores han cuestionado la seguridad de esta técnica (2). No obstante, también se 
han documentado casos de neumoencéfalo tras anestesia subaracnoidea y punciones lumbares diagnósticas o terapéuticas $(3,4,5)$.

El espectro clínico del neumoencéfalo es muy variable, oscilando entre la ausencia de síntomas y el cortejo sintomático de hipertensión intracraneal. Son síntomas frecuentes la cefalea y las alteraciones del nivel de conciencia. En la mayor parte de casos el tratamiento es conservador, con observación clínica y radiológica (6).

En el caso que nos ocupa, lo llamativo de la imagen radiológica inicial hizo que el cuadro fuera atribuido de forma precipitada a una complicación de la anestesia a pesar de que, como confirmó la TAC de control, la clínica orientaba más hacia el origen isquémico que al neumoencéfalo como causa del cuadro.

No parece que el neumoencéfalo en este caso haya tenido más relevancia que el de un hallazgo casual asintomático, ya que tanto los síntomas iniciales como la evolución de la paciente pueden ser explicadas en el contexto del ictus isquémico. Tampoco se encuentra relación causal entre el neumoencéfalo $\mathrm{y}$ el ictus puesto que se presentaron en territorios cerebrales diferentes y alejados entre sí.

Pese a que el anestesista responsable no reportó incidentes en el informe anestésico, la técnica anestésica sigue siendo la explicación más probable del neumoencéfalo, ya sea por una punción dural inadvertida durante la técnica de localización del espacio epidural, o bien por la inyección accidental de aire durante la técnica intradural.

A pesar de que la evidencia científica y la experiencia respaldan ampliamente la seguridad de las técnicas neuroaxiales de anestesia, el temor a complicaciones neurológicas derivadas de las mismas forma parte de la cultura popular. Ante un evento de estas características, es imprescindible realizar una evaluación cuidadosa del mismo, valorando todas las posibles causas antes de etiquetarlo como yatrogénico.

Aunque nuestra paciente y su familia recibieron información clara y veraz durante todo el proceso, ella se fue a su domicilio convencida de haber sido víctima de una complicación de la anestesia.

\section{Bibliografía}

1. Brull R, McCartney C, Chan V, El-Beheiry H. Neurological complications after regional anesthesia: Contemporary estimates of risk. Anesth Analg. 2007; 104(4): 965-974. (PubMed)

2. Arango G, Arango MF, Restrepo CE. ¿Se justifica seguir enseñando pérdida de resistencia utilizando aire en lugar de suero salino para identificar el espacio epidural? A propósito de un caso de neumoencéfalo. Rev Soc Esp Dolor. 2002; 9:405-408. (

3. Avellanal M, Olmedilla L, Ojea R, Rueda ML, Navia J. Pneumocephalus after Spinal Anesthesia Anesthesiology 1996; 85: 423-425. ( $\underline{\text { HTML) }}$

4. Kozikowsky GP, Cohen SP. Lumbar puncture associated with pneumocephalus: Report of a case. Anesth Analg 2004; 98(2): 524-6. (PubMed)

5. Roderick L, Moore DC, Artru AA. Pneumocephalus with headache during spinal anesthesia. Anesthesiology. 1985; 62(5):690-2. (PubMed)

6. Perez Delgado FJ, Jacó Batista N. Neumoencéfalo. Med Gen y Fam. 2016; 5(2):56-58. 
Correspondencia al autor

Belén Bardisa de la Iglesia

Aceptado para blog en mayo de 2018.

belenbardisa@gmail.com

Adjunta Servicio de Anestesiología y

Reanimación.

Hospital Francesc de Borja. Gandia, Valencia. 